\title{
Breast-feeding mothers can exercise: results of a cohort study
}

\author{
Dada Su', Yun Zhao ${ }^{1}$, Colin Binns ${ }^{1, *}$, Jane Scott ${ }^{2}$ and Wendy Oddy ${ }^{1}$ \\ ${ }^{1}$ School of Public Health, Curtin University, GPO Box U1987, Perth, Western Australia 6845, Australia: \\ ${ }^{2}$ Division of Developmental Medicine, Human Nutrition Section, University of Glasgow, Glasgow, UK
}

Submitted 13 July 2006: Accepted 13 December 2006: First published online 22 May 2007

\begin{abstract}
Objectives: To study the relationship between exercise by the mother and breastfeeding initiation and duration, and its effect on infant growth.

Design: A cohort study of mothers and infants, recruited at birth. Infant feeding methods were recorded in detail and breast-feeding was categorised as 'any' or 'full'. Exercise levels were categorised using the metabolic equivalent tasks approach based on details of physical activity recorded in questionnaires.

Setting: Perth, Western Australia.

Subjects: A total of 587 mothers were interviewed on seven occasions over a period of 12 months.

Results: There was no difference in the means of infant weight and length changes, indicating that exercise appeared to have no significant influence on infant growth up to 52 weeks after birth $(P=0.236$ and 0.974 , respectively). The mother's level of exercise was not significantly associated with breast-feeding to 6 or 12 months. This applied to 'full' and 'any' categories of breast-feeding.

Conclusion: Exercise does not affect breast-feeding outcomes at the usual levels of activity undertaken by mothers. Breast-feeding and exercise are important for maintaining and promoting health, and this study provides reassurance to health professionals wishing to encourage mothers to continue both behaviours.
\end{abstract}

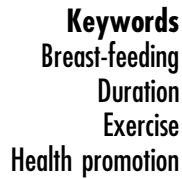

Exercise is an important part of health-promotion programmes because of its documented benefits in promoting health at all ages. In the nutrition field, the benefits of breast-feeding receive as much emphasis as exercise in general health promotion ${ }^{1,2}$.

There are numerous benefits of breast-feeding to the infant and the mother, which have been reviewed extensively ${ }^{1,3,4}$. Exercise brings many health benefits for cardiovascular and musculoskeletal health ${ }^{5,6}$. Energy expenditure provides the essential element in daily individual weight balance and recent research has shown the importance of exercise in the prevention of cancer ${ }^{7,8}$.

Because of the benefits of breast-feeding and of exercise, it is natural for mothers to want to do both. The effect of exercise on breast-milk composition and volume were reviewed by Clapp and Little in 1995, who noted that there had been occasional reports in the literature that physical activity had an adverse impact on breast-feeding performance ${ }^{9}$. Most concerns related to an increase in lactic acid in breast milk following exercise and its effect on taste to infants. However, subsequent studies have demonstrated that the quantity and quality of milk and infant growth are not adversely affected by moderate maternal exercise ${ }^{10,11}$. Where the mother's diet is adequate, exercise has been shown not to affect the long-chain polyunsaturated acid content of breast milk ${ }^{12}$.
However it is a commonly held belief amongst mothers that exercise while breast-feeding can affect the taste of breast milk and this may affect their infant's health. A literature search has not revealed any previous studies of breast-feeding and exercise in Australia.

An important question for mothers is 'Can I continue (or recommence) to exercise and breast-feed?' Mothers today have been educated about the overwhelmingly positive benefits of breast-feeding for their infants and at the same time they wish to exercise to regain their pre-pregnancy fitness level. Thus the objectives of the present study were to document the relationships between exercise by the mother and breast-feeding initiation and duration, and the effect on infant growth.

\section{Methods}

The second Perth Infant Feeding Study (PIFS II) was conducted between 2002 and 2004. Mothers were recruited from women delivering at two public hospitals in Perth, Western Australia. Women were eligible for the study if they had delivered a live infant free of serious health problems requiring transfer to Perth's major maternity hospital for critical care ${ }^{13}$. A total of 870 mothers of newborn infants were contacted and 587 completed the baseline questionnaire while they were in hospital on their 
infant feeding practices and preferences. This represented $68 \%$ of the eligible mothers contacted; a high response rate for this type of survey, reducing the likelihood of a response bias. The mothers in the study were interviewed on seven occasions at regular intervals until their infants reached 12 months of age. There were no significant differences in the age and level of education of participants and non-participants, indicating that participants were representative of the population from which they were drawn ${ }^{13}$. The categories of breast-feeding used in this study were 'any breast-feeding' and 'full breastfeeding' ${ }^{14}$. 'Full breast-feeding' includes infants who received only breast milk or breast milk plus small amounts of water, water-based drinks or fruit juice.

There are many ways in which exercise can be measured. Some are very restrictive such as the use of whole-body calorimetry, while modern studies use doubly labelled water, which is accurate and unobtrusive ${ }^{15}$. However this method has the disadvantage of high cost and in this study the large sample size precluded its use. For that reason exercise was measured with a comprehensive questionnaire using the metabolic equivalent task (MET) approach. Logging physical activity and multiplying the nature and duration of these activities by their metabolic equivalents has been widely used to estimate activity thermogenesis ${ }^{15}$.

Physical activity was assessed in terms of type, intensity and duration. The type of exercise included all occupational and leisure-time activities. Intensity was categorised as light, moderate (e.g. cycling on level ground, gardening, housework, cooking, walking, tai chi) or vigorous (e.g. moving heavy furniture, loading or unloading trucks, jogging, cycling up hill, swimming). The classification of tasks was based on the amount of energy a person expends in performing the activity. The questions used were developed for the Hawaiian Cancer Study ${ }^{16}$ and the National Health Survey ${ }^{17}$ and have been used in previous studies $^{7,8}$.

To measure duration, the weekly average time spent in each activity level was recorded. The overall physical activity exposure was then quantified in terms of MET, representing the number of kilocalories per hour each kilogram of body weight expended in activities. For this study energy expenditure was examined in quartiles in the period since the previous interview and then summed over the 6- or 12-month period.

The project was approved by the research ethics committees of the two participating hospitals and by the Human Research Ethics Committee of Curtin University. All mothers were given an information sheet about the study and those willing to participate gave their signed informed consent before being asked to complete the questionnaires.

The data were coded and analysed using the SPSS for Windows program (SPSS Inc.). Initially descriptive statistics were computed and differences between MET groups were examined using $\chi^{2}$ analysis. Cox regression analysis controlling for a variety of factors known to be associated with breast-feeding duration was used to model the time-to-event data and to determine which variables had an independent effect on the duration of breastfeeding ('any' and 'full') for those mothers who were breast-feeding at the time of discharge from hospital.

\section{Results}

The demographic factors of mothers and infants that might be expected to have an influence on undertaking exercise at a selected time point, week 4 , are listed in Table 1 . This time period was chosen as mothers would be unlikely to return to exercise before this time. The numbers and percentage of mothers who exercised at the four levels of MET-hours per week are given for each variable. The analysis of demographic variables showed that mother's parity $(P=0.000)$ and infant birth weight $(P=0.013)$ were significantly associated with undertaking more exercise at week 4. The relationships between demographic factors and undertaking exercise were calculated for other time points (before pregnancy, week 10, 16, 22, 32, 40 and 52) and were similar to the results at week 4.

\section{Exercise and breast-fed infant weight and length}

Infant weight and length were used as proxy measures to assess the adequacy of breast-milk production. Nonparametric tests were used to explore the influence of exercise on infant weight and length. The means of weight and length changes between week 4 and week 52 of infants whose mothers were breast-feeding by each intensity level activity were compared. Figure 1 is a boxand-whisker plot of weight gain, with the box representing the interquartile range and the whiskers the range. There was no difference in the means of infant weight and length changes, indicating that exercise appeared to have no significant influence on infant growth up to 52 weeks after birth $(P=0.236$ and 0.974 , respectively).

\section{Influence of exercise on breast-feeding}

The duration of 'any breast-feeding' and 'full breastfeeding' up to 12 months was estimated using the KaplanMeier survival method for different exercise levels (quartiles). The exercise intensity levels were measured by total MET-hours in a year (see Table 2). There was no difference in the duration of 'any' or 'fully' breast-feeding between each level of exercise intensity $(P=0.1275$ and 0.2092 , respectively). In addition, the determinants of duration of 'any breast-feeding' and 'full breast-feeding' to 6 months were explored with multivariate analysis using Cox regression and the forward selection procedure. A summary of the results obtained from the Cox regression of 'any breast-feeding' to 6 months is shown in Table 2 . Details of the variables associated with breast-feeding duration to 6 months have been published previously and in the current paper only detailed results for the effect of exercise are presented ${ }^{18,19}$. 
Table 1 Demographic variables and exercise at week $4(n=587)$

\begin{tabular}{|c|c|c|c|c|c|}
\hline & \multicolumn{4}{|c|}{ Total MET-hours grouped at week 4} & \multirow[b]{2}{*}{ Total } \\
\hline & $0-15$ & $16-28$ & $29-47$ & $48+$ & \\
\hline \multicolumn{6}{|l|}{ Age (years) } \\
\hline$<20$ & $7(31.8)$ & $3(13.6)$ & $8(36.4)$ & $4(18.2)$ & $22(100)$ \\
\hline $20-29$ & 77 (30.3) & 49 (19.3) & $73(28.7)$ & $55(21.7)$ & $254(100)$ \\
\hline $30+$ & $64(27.7)$ & $53(22.9)$ & $67(29.0)$ & 47 (20.3) & $231(100)$ \\
\hline \multicolumn{6}{|l|}{ Education (years) } \\
\hline$<12$ & $52(24.9)$ & $43(20.6)$ & $64(30.6)$ & $50(23.9)$ & $209(100)$ \\
\hline 12 & $44(31.4)$ & $26(18.6)$ & $42(30.0)$ & $28(20.0)$ & $140(100)$ \\
\hline$>12$ & 49 (32.2) & $35(23.0)$ & $41(27.0)$ & $27(17.8)$ & $52(100)$ \\
\hline \multicolumn{6}{|l|}{ Parity $^{*}$} \\
\hline Primiparous & $76(39.6)$ & $38(19.8)$ & $55(28.6)$ & $23(12.0)$ & $192(100)$ \\
\hline Multiparous & $72(22.9)$ & $67(21.3)$ & $93(29.5)$ & $83(26.3)$ & $315(100)$ \\
\hline \multicolumn{6}{|l|}{ Birth weight $(g)^{*}$} \\
\hline$<2500$ & $3(30.0)$ & $6(60.0)$ & $1(10.0)$ & $0(0)$ & $10(100)$ \\
\hline $2500+$ & $144(29.3)$ & 99 (20.2) & $146(29.7)$ & $102(20.8)$ & $491(100)$ \\
\hline \multicolumn{6}{|l|}{ Mother's country of birth } \\
\hline Australia/New Zealand & $103(27.8)$ & $73(19.7)$ & $119(32.1)$ & $76(20.5)$ & $371(100)$ \\
\hline UK/Ireland & $13(29.5)$ & $11(25.0)$ & 9 (20.5) & $11(25.0)$ & $44(100)$ \\
\hline Other & $32(35.2)$ & $20(22.0)$ & $20(22.0)$ & $19(20.9)$ & $91(100)$ \\
\hline \multicolumn{6}{|l|}{ Income level (A\$) } \\
\hline$<15000$ & 37 (33.3) & $19(17.1)$ & $31(27.9)$ & $24(21.6)$ & $111(100)$ \\
\hline $15001-25000$ & $44(27.8)$ & $33(20.9)$ & $47(29.7)$ & $34(21.5)$ & $158(100)$ \\
\hline $25001-40000$ & $27(29.0)$ & $18(19.4)$ & $27(29.0)$ & $21(22.6)$ & $93(100)$ \\
\hline $40000+$ & $37(26.6)$ & $33(23.7)$ & $42(30.2)$ & $27(19.4)$ & 139 (100) \\
\hline
\end{tabular}

MET - metabolic equivalent task.

Data are presented as $n(\%)$.

* Significant association between the variables parity $(P=0.000)$ and birth weight $(P=0.013)$ with exercise.

The analysis was repeated for 'full breast-feeding' duration up to 6 months and again for 'any breast-feeding' to 12 months, as $22 \%$ of the mothers in this study were still breast-feeding at 1 year. The amount of exercise undertaken by the mothers was not associated with 'any breast-feeding' or 'full breast-feeding' duration up to 6 months or up to 12 months. Specifically, mothers who had different levels of energy expenditure (MET-hours) were not found to be statistically different in their duration of any breast-feeding.

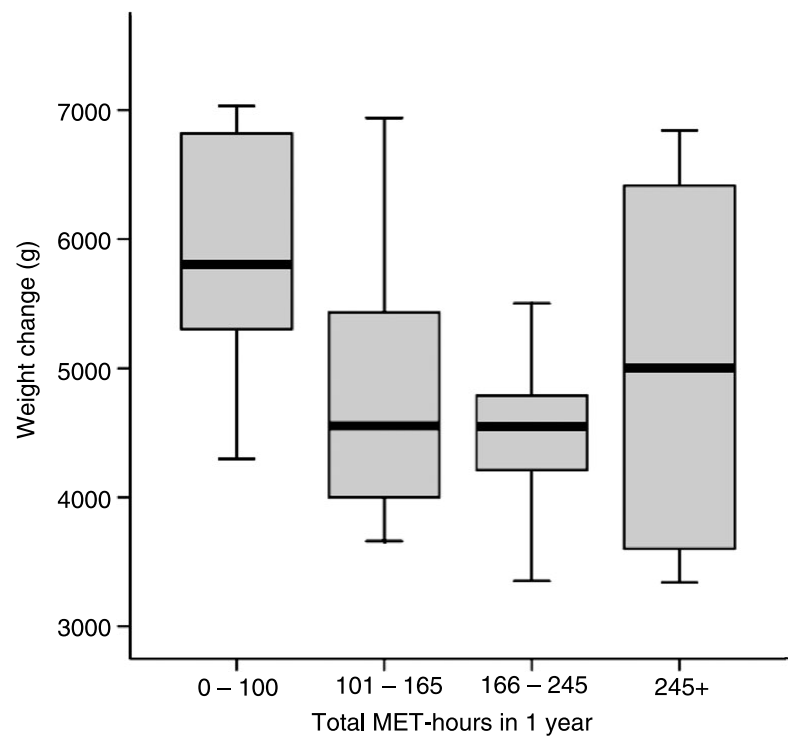

Fig. 1 Infant weight change during the first year of life by maternal exercise in the Perth Infant Feeding Study Mark II (MET - metabolic equivalent task)

\section{Discussion}

Breast-feeding and exercise are both of major health interest to parents, particularly mothers. This analysis of data from the PIFS II indicated that there was no evidence to support the claim that exercise, especially moderate exercise, would have a negative impact on breast-feeding. None of the mothers in this study consistently reached levels of energy activity that would be associated with elite international athletes, so no information is available on the effects of extreme energy expenditure on breast-feeding performance.

\section{Effect of exercise on infant growth}

The study demonstrated that the level of exercise by breastfeeding mothers does not affect the growth of their infants in terms of weight and length. The lack of an impact on growth in breast-fed infants is a good indication that exercise has no effect on the quantity and quality of breastmilk production. Our results support those reported by Lovelady et al., who compared eight well-nourished exercising and eight sedentary women whose infants were 9-24 weeks old and exclusively breast-fed ${ }^{20}$. They demonstrated there was no difference between the groups in plasma hormones, milk energy, lipid, protein or lactose content. In addition they confirmed that milk volume was slightly though not significantly higher in the exercise group (839 vs. $776 \mathrm{~g} \mathrm{day}^{-1}$ ).

Some previous studies showed that lactic acid seemed to increase in the breast milk of women exercising at 
Table 2 Association of exercise with the risk of discontinuing 'any breast-feeding' before 6 months, before and after adjustment for potential confounders*, in the Perth Infant Feeding Study Mark II

\begin{tabular}{lcccc}
\hline Total MET-hours in 1 year & Crude RR & $95 \% \mathrm{Cl}$ & Adjusted RR $¥$ ‡ & $95 \% \mathrm{Cl}$ \\
\hline $0-100$ & 1.000 & & 1.000 & \\
$101-165$ & 0.665 & $0.465-0.952$ & 0.719 & $0.473-1.093$ \\
$166-245$ & 0.787 & $0.555-1.117$ & 0.840 & $0.550-1.281$ \\
$245+$ & 0.807 & $0.571-1.140$ & 0.947 & $0.628-1.428$ \\
\hline
\end{tabular}

MET - metabolic equivalent task; RR - relative risk; $\mathrm{Cl}$ - confidence interval.

-2 log likelihood (deviance) $=2056.418, \mathrm{df}=16$.

* The variables controlled for in the model were infant sex, infant birth weight, infant admitted to a special-care nursery, maternal marital status, demand feeding, parity, attendance at antenatal classes, maternal age, maternal infant feeding attitude score, maternal occupation, age of infant when mother returned to work, maternal years of education, maternal country of birth, how many times mother had been sick in one year, maternal grandmother's feeding preference, father's feeding preference, age by which dummy was introduced, time at which infant feeding method decided, how many times mother had breast-feeding problems in one year, and total MET-hours spent for exercise in one year.

$\dagger$ Non-significant variables were sex, infant admitted to a special-care nursery, maternal marital status, demand feeding, parity, attendance at antenatal classes, maternal occupation, maternal years of education, maternal country of birth, how many times mother had been sick in one year, and total MET-hours spent for exercise in one year.

$\ddagger$ All variables in the final model were variables for which, when excluded, the change in deviance compared with the corresponding $x^{2}$ test statistic on the relevant degrees of freedom was significant.

maximal intensity ${ }^{21}$. Controversy exists as to whether this short-term increase in lactic acid makes the breast milk less acceptable to the nursing infant ${ }^{11,21}$. While we did not directly measure lactic acid or any other markers of taste in breast milk, our finding that maternal exercise was not associated with either infant growth or breast-feeding duration would suggest that maternal exercise had no adverse effect on the taste of breast milk.

We could locate no published papers on cohort studies of the association between exercise and duration of breastfeeding. The present study has indicated that breastfeeding duration up to 6 and 12 months was not adversely affected by exercise, regardless of the intensity. Studies that include the duration of breast-feeding as a variable show that there is an increasing dose-response in health benefits, i.e. the longer the duration of breast-feeding, the more health benefits are accrued by both mothers and infants $^{22-24}$. The finding of the lack of a relationship between exercise and duration of breast-feeding in the current study may help to reduce the socio-cultural inhibition of extended breast-feeding to postpartum exercise for breast-feeding women.

It is rare that studies with negative findings are so significant for health promotion. In this case, the finding that mothers can exercise and continue to breast-feed is a significant finding. This should be the basis of health-promotion programmes to mothers and fathers. Fathers have an important role to play in caring for infants while their partner exercises or by accompanying the mother. Modern technology (improved exercise baby carriages) allows mothers to walk and jog with their infants in safety.

There are several limitations that need to be taken into account when considering the results of this study. None of the mothers was exercising at the level of an elite athlete, and hence the results may not apply to extreme levels of exercise. The exercise levels were measured by a standardised questionnaire and no independent measures of energy expenditure are available, such as metabolic studies using doubly labelled water. This study included mothers recruited from public hospitals and therefore cannot be considered to be representative of more affluent populations. However, these mothers are representative of the 'hard to reach' groups in health-promotion programmes. Therefore the lessons learned from this study could be appropriately applied in health-promotion programmes. The message you can exercise and breast-feed' should be communicated to all mothers and mothers-to-be.

\section{Conclusion}

In the past it has been common practice to advise mothers to restrict the amount of exercise they do while breastfeeding, as it would affect the taste of their breast milk and overall breast-milk production. This study shows that mothers can exercise without having any impact on breast-feeding performance. This is important news for all mothers and for all health professionals who communicate health messages to mothers.

\section{Acknowledgements}

Sources of funding: The Perth Infant Feeding Study was funded by the Australian Department of Health and Ageing. The project was initiated by C.B., the principal investigator.

Conflict of interest declaration: The authors declare that they have no conflicts of interest.

Authorship responsibilities: D.S. - data collection, data analysis, paper writing; Y.Z. - statistical analysis; C.B. - chief investigator, project design, data analysis, paper writing; J.S. - associate investigator, data management, paper writing; W.O. - associate investigator, paper writing.

Acknowledgements: We thank the mothers, hospitals, nursing staff and medical practitioners who assisted in the study. Without their assistance the study would not have been possible. 


\section{References}

1 Binns C, Davidson G. Infant feeding guidelines for health workers. In: Dietary Guidelines for Children in Australia. Canberra: National Health and Medical Research Council, 2003.

2 National Health and Medical Research Council. Food for Health: Dietary Guidelines for Australians - A Guide to Healthy Eating. Canberra: National Health and Medical Research Council, 2003.

3 Binns C. Encourage and support breastfeeding. In: Dietary Guidelines for Children and Adolescents in Australia. Canberra: National Health and Medical Research Council, 2003; 6.

4 American Academy of Pediatrics. Breastfeeding and the use of human milk. Pediatrics 2005; 115(2): 496-505.

5 Centers for Disease Control and Prevention (CDC). Physical Activity and Health: A Report to the Surgeon General. Washington, DC: CDC, US Department of Health and Family Services, 1996.

6 Shaw J. The deadliest sin: the benefits of exercise and the dangers of sloth. Harvard Magazine 2004; (March): 37-43.

7 Zhang M, Lee A, Binns C. Physical activity and epithelial ovarian cancer risk: a case-control study in China. International Journal of Cancer 2003; 105(6): 838-43.

8 Jian L, Shen ZJ, Lee AH, Binns CW. Moderate physical activity and prostate cancer risk: a case-control study in China. European Journal of Epidemiology 2005; 20(2): 155-60.

9 Clapp J, Little K. The interaction between regular exercise and selected aspects of women's health. American Journal of Obstetrics and Gynecology 1995; 173(1): 2-9.

10 Dewey K, Lovelady C, Nommsen-Rivers L, McCrory M, Lonnerdal B. A randomized study of the effects of aerobic exercise by lactating women on breast-milk volume and composition. New England Journal of Medicine 1994; 330(7): 449-53.

11 Wright KS, Quinn TJ, Carey GB. Infant acceptance of breast milk after maternal exercise. Pediatrics 2002; 109(4): 585-9.

12 Bopp M, Lovelady C, Hunter C, Kinsella T. Maternal diet and exercise: effects on long-chain polyunsaturated fatty acid concentrations in breast milk. Journal of the American Dietetic Association 2005; 105(7): 1098-103.

13 Graham KI, Scott JA, Binns CW, Oddy WH. National targets for breastfeeding at hospital discharge have been achieved in Perth. Acta Paediatrica 2005; 94(3): 352-6.

14 Webb K, Marks GC, Lund-Adams M, Rutishauser I, Abraham B. Towards a National System for Monitoring Breastfeeding in Australia: Recommendations for Population Indicators, Definitions and Next Steps. Canberra: Commonwealth Department of Health and Aged Care, 2001.

15 Levine JA. Measurement of energy expenditure. Public Health Nutrition 2005; 8(7A): 1123-32.

16 Goodman MT, Hankin JH, Wilkens LR, Lyu LC, McDuffie K, Liu LQ, et al. Diet, body size, physical activity, and the risk of endometrial cancer. Cancer Research 1997; 57(22): 5077-85.

17 Australian Bureau of Statistics (ABS). 1995 National Health Survey: Users' Guide. Catalogue No. 4263.0. Canberra: ABS, 1996.

18 Scott JA, Binns CW, Oddy WH, Graham KI. Predictors of breastfeeding duration: evidence from a cohort study. Pediatrics 2006; 117(4): e646-55.

19 Scott JA, Binns CW, Graham KI, Oddy WH. Temporal changes in the determinants of breastfeeding initiation. Birth 2006; 33(1): 37-45.

20 Lovelady CA, Lonnerdal B, Dewey KG. Lactation performance of exercising women. American Journal of Clinical Nutrition 1990; 52(1): 103-9.

21 Wallace JP, Inbar G, Ernsthausen K. Infant acceptance of postexercise breastmilk. Pediatrics 1992; 89(6): 1245-7.

22 Finch C, Daniel E. Breastfeeding education program with incentives increases exclusive breastfeeding among urban WIC participants. JAMA: Journal of the American Medical Association 2002; 102(7): 981-4.

23 Dettwyler K. A time to wean. In: Stuart-Macadam P, Dettwyler K, eds. Breastfeeding: Biocultural Perspectives. New York: Aldine de Gruytyer, 1995; 39-73.

24 United Nations Children's Fund (UNICEF). Panel 13: breastfeeding breakthroughs. In: State of the World's Children. New York: UNICEF, 1998; 50. 